\title{
22. A Chromosome Study of the Congenital Defectives and their Relatives seeking Genetic Advices
}

\author{
By Tetsuji Kadotani,*) Yoko Watanabe,*) Takashi Katano,**) \\ and Ichiro TAKEMURA***) \\ (Communicated by Sajiro Makino, M. J. A., April 12, 1982)
}

This report presents a summary of the direct combination-work undertaken between genetic counselors and cytogeneticists. During a period from November 1975 to the end of December 1981, 515 cases were collected for chromosomal researches. This article is a product of the collaboration-work with the institutions for the congenital defectives and the Kadotani Medical Research Foundation. The 515 cases under study consisted of 205 cases from the Hiroshima City Welfare Center for the Handicapped Children's Center, ${ }^{\dagger)} 92$ cases from the Kojika-Gakuen, ${ }^{\dagger+}$ and 218 cases from the Kadotani Medical Research Foundation.

The chromosome studies were performed with the standard blood cultures. The chromosomes were analysed following the conventional Giemsa, the G- and Q-banding procedures.

Results and remarks. Four hundred and nine out of the 515 cases herein concerned were congenital defectives and the remaining 106 cases were their relatives. Three hundred and thirteen out of 409 cases with congenital defects were found to possess a normal karyotype, leaving 96 cases showing abnormal karyotypes, being $23.5 \%$ in incidence. Among the abnormalities, 7 out of 96 cases were found to be transmitted through the parental line being $7.3 \%$ in incidence, while the remaining 89 cases seemed to be of sporadic origin, since the parents of those cases were chromosomally normal. Out of 96 cases with chromosome abnormalities, 84 cases showed autcsomal abnormalities, while the remaining 12 cases had sexchromosome abnormalities. The data so far obtained are summarized in Table I, and critical details for 15 cases of them are referred to in the literature listed (1-15). Chromosome abnormalities of 81 cases

*) The Kadotani Medical Research Foundation, 1248, Saijohigashi, Saijo, Higashihiroshima, Japan.

**) The Hiroshima City Welfare Center for the Handicapped Children's Center, Hikari-machi, Hiroshima, Japan.

***) The Kojika-Gakuen, 4901, Awayacho, Miyoshi, Hiroshima, Japan.

†) Katano et al.: Proc. Japan Acad., vol. 54B, pp. 111-115, 1978; vol. 56B, pp. 263-267, 1980 .

††) Kadotani et al.: Proc. Japan Acad., vol. 56B, pp. 431-436, 1980. 
Table I. Chromosome abnormalities of 96 cases found in 515 cases seeking genetic advices

\begin{tabular}{|c|c|c|c|c|c|}
\hline Autosomal abnormality & $\begin{array}{c}\text { No. } \\
\text { of } \\
\text { cases }\end{array}$ & Ref. & $\begin{array}{l}\text { Sex-chromosome } \\
\text { abnormality }\end{array}$ & $\begin{array}{c}\text { No. } \\
\text { of } \\
\text { cases }\end{array}$ & Ref. \\
\hline $46, \mathrm{XX}, \operatorname{dir} \operatorname{dup}(3)(\mathrm{q} 22 \rightarrow \mathrm{q} 27)$ & 1 & 1) & $45, \mathrm{X} / 46, \mathrm{Xr}(\mathrm{X}) *$ & 1 & \\
\hline $\begin{array}{l}\text { 46,XY,der(1),ins }(1 ; 9) \\
\text { (q11;q12q13)mat }\end{array}$ & 1 & 2) & $\begin{array}{l}45, \mathrm{X}^{*} \\
48, \mathrm{XXXY}^{*}\end{array}$ & $\begin{array}{l}1 \\
1\end{array}$ & \\
\hline $\begin{array}{l}\text { 46,XY,1qh+,rcp }(3 ; 4) \\
\text { (q23;p14)* }\end{array}$ & 1 & & $45, \mathrm{X} / 46, \mathrm{XX}^{*}$ & 1 & \\
\hline $46, \mathrm{XX}$ or $\mathrm{XY}, 1 \mathrm{qh}+*$ & 7 & & $45, \mathrm{X} / 47, \mathrm{XXX}$ & 1 & 13) \\
\hline $46, X Y, 5 p-*$ & 1 & & $\begin{array}{l}\text { 46,XX,der(9),ins }(9 ; \mathrm{X}) \\
\text { (q13;q22q24)mat }\end{array}$ & 1 & 14) \\
\hline $46, X Y, 5 p+*$ & 1 & & $47, \mathrm{XXX}^{*}$ & 1 & \\
\hline $\begin{array}{l}\text { 46,XY,der(5),rcp(5;17) } \\
\text { (p13;q23)mat }\end{array}$ & 1 & 3) & $46, \mathrm{XY}$ (female)* & 3 & \\
\hline $46, X Y, \operatorname{del}(5)(q 15 q 31)$ & 1 & 4) & $\begin{array}{l}\text { 46,XX, der(1), ins }(1 ; \mathrm{Y}) \\
\text { (q21;q11)pat }\end{array}$ & 1 & 15) \\
\hline $46, X Y, \operatorname{del}(5)(q 22 q 31)^{*}$ & 1 & & $46, \mathrm{XYp}+*$ & 1 & \\
\hline $46, \mathrm{XX} / 46, \mathrm{XX}, \mathrm{r}(9)(\mathrm{p} 24 \mathrm{q} 34)$ & 1 & 5) & & & \\
\hline$\underset{\text { mat }}{46, \mathrm{XX}}, \operatorname{der}(9), \mathrm{t}(4 ; 9)(\mathrm{q} 23 ; \mathrm{p} 24)$ & 1 & 6) & & & \\
\hline $46, X Y, \operatorname{inv}(9)(p 11 q 13)$ pat & 1 & 7) & & & \\
\hline $46, X Y, r c p(7 ; 14)(q 11 q 22)^{*}$ & 1 & & & & \\
\hline $46, \mathrm{XX}, 9 \mathrm{qh}+*$ & 1 & & & & \\
\hline $\begin{array}{l}\text { 46,XX,der(15),ins(15;3) } \\
\text { (p13;q22)mat }\end{array}$ & 1 & 8) & & & \\
\hline $47, \mathrm{XX},+\mathrm{E}^{*}$ & 1 & & & & \\
\hline $47, \mathrm{XX},+18^{*}$ & 2 & & & & \\
\hline $46, \mathrm{XX}, \operatorname{del}(18)(\mathrm{p} 1105)$ & 1 & 9) & & & \\
\hline $46, \mathrm{XX}, \mathrm{r}(18)(\mathrm{p} 11 ; \mathrm{q} 23)^{*}$ & 1 & & & & \\
\hline $47, \mathrm{XX}$ or $\mathrm{XY},+21^{*}$ & 51 & & & & \\
\hline $47, \mathrm{XY},+22$ & 1 & 10) & & & \\
\hline $47, \mathrm{XY},+\operatorname{del}(22)(\mathrm{q} 13)$ & 1 & 11) & & & \\
\hline $47, \mathrm{XX},+\operatorname{del}(22)(\mathrm{q} 13) \mathrm{mat}$ & 1 & 12) & & & \\
\hline $46, \mathrm{XX},-21,+\mathrm{Ph}^{1 *}$ & 1 & & & & \\
\hline $46, \mathrm{XY},-21, \mathrm{t}(21 ; 21)^{*}$ & 1 & & & & \\
\hline $46, \mathrm{XX},-21, \mathrm{t}(14 ; 21)^{*}$ & 2 & & & & \\
\hline Total & 84 & & Total & 12 & \\
\hline
\end{tabular}

marked with asteriscs in Table I have not been described by us. Some examples of chromosome aberrations are shown in Fig. 1. Details of some of these abnormal cases will be published elsewhere in the near future.

Then it is apparent that the chromosome study serves as an important tool for the clinical diagnosis of the defectives and their relatives seeking genetic advices.

Acknowledgements. We are much obliged to Emeritus Professor Sajiro Makino, M. J. A., the research director of the Kadotani 
A

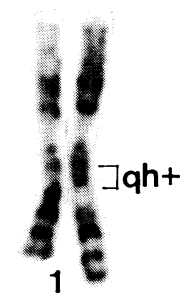

B

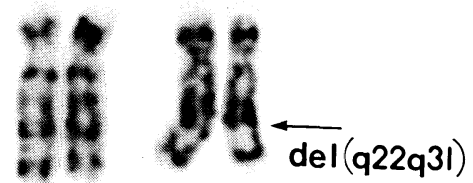

4

D

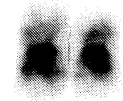

16

$\mathbf{E}$

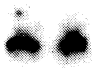

21

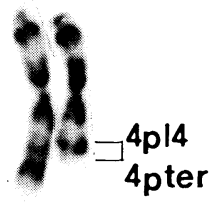

3

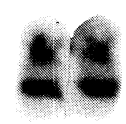

17

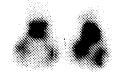

22

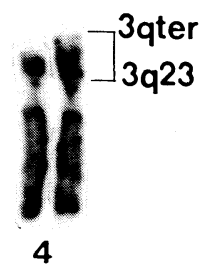

C

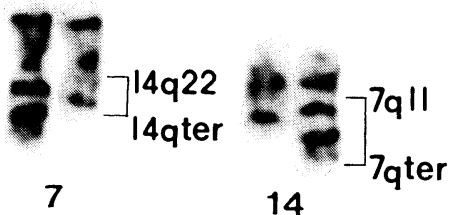

14

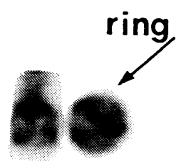

18

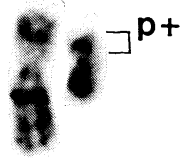

$X Y$

Fig. 1. G-banding partial karyotypes of some of abnormal cases which have not been published. A: 46,XY,1qh+,rcp(3;4)(q23;p14). B: 46,XY, $\operatorname{del}(5)(q 22 q 31) . \quad$ C: 46,XY,rcp $(7 ; 14)(q 11 q 22) . \quad$ D: 46,XX,r(18)(p11;q23). E: $46, \mathrm{XYp}+$.

Medical Research Foundation, for improvement of this manuscript with invaluable advice. And also our thanks are due to Drs. Motochiyo Murakami, Morihisa Funatsu, Shigeki Takeuchi, Takashi Yokoyama, Hideki Yamaoka, Akihiko Shiote, and Suzue Kanata for their clinical examinations. Financial aid from the Japan Academy is gratefully acknowledged here.

\section{References}

1) Kadotani, T., Watanabe, Y., Kiyuna, T., Kawamoto, T., and Takemura, I. (1979) : Proc. Japan Acad., 55B, 431-435.

2) - (1980) : ibid., 56B, 157-161. 
[Vol. 58(B),

3) Kadotani, T., Hiyama, Y., Kawakami, M., Ohara, H., and Makino, S. (1977) : Proc. Japan Acad., 53, 155-158.

4) Kadotani, T., Katano, T., Murakami, M., and Watanabe, Y. (1979) : ibid., 55B, 300-304.

5) Kadotani, T., Watanabe, Y., Kiyuna, T., Kawamoto, T., Takemura, I., and Funatsu, M. (1980) : ibid., 56B, 85-88.

6) Kadotani, T., Watanabe, Y., Kiyuna, T., and Takeuchi, S. (1981) : ibid., 57B, 374-377.

7) (1981): ibid., 57B, 378-380.

8) Kadotani, T., Watanabe, Y., Kiyuna, T., Kawamoto, T., Katano, T., and Takemura, I. (1980) : ibid., 56B, 621-626.

9) Kadotani, T., Watanabe, Y., Araune, M., Nakajima, K., Yokoyama, T., and Ishii, T. (1978) : ibid., 54B, 607-611.

10) Kadotani, T., Katano, T., Yamaoka, H., Murakami, M., Nakamoto, Y., and Watanabe, Y. (1978) : ibid., 54B, 163-166.

11) - (1978): ibid., 54B, 217-221.

12) Kadotani, T., Katano, T., Murakami, M., and Shiote, A. (1978) : ibid., 54B, 623-627.

13) Kadotani, T., Katano, T., Murakami, M., and Watanabe, Y. (1980) : Chromosome Information Service, 28, 20-21.

14) Kadotani, T., Katano, T., Takeuchi, S., Murakami, M., and Watanabe, Y. (1978) : Proc. Japan Acad., 54B, 538-541.

15) Kadotani, T., Watanabe, Y., Kiyuna, T., Kawamoto, T., and Katano, T. (1980): ibid., 56B, 616-620. 